\title{
Feedback linearization and simultaneous stiffness-position control of robots with antagonistic actuated joints
}

\author{
G. Palli and C. Melchiorri \\ DEIS, Dept. of Electronics, Computer Science and Systems \\ University of Bologna \\ Via Risorgimento 2, 40136 Bologna, Italy \\ \{gpalli,cmelchiorri\}@deis.unibo.it
}

\author{
T. Wimböck, M. Grebenstein and G. Hirzinger \\ Institute of Robotics and Mechatronics \\ German Aerospace Center (DLR) \\ Oberpfaffenhofen, 82234 Wessling, Germany \\ \{thomas.wimboeck, markus.grebenstein, gerd.hirzinger\}@dlr.de
}

\begin{abstract}
In this paper, the dynamic model of a robot with antagonistic actuated joints is presented, and the problem of full linearization via static state feedback is analyzed. The use of transmission elements with nonlinear relation between the displacement and the actuated force allows to control both the position and the stiffness of each joint. The main advantage of this actuation modality is that the achieved stiffness becomes a mechanical characteristic of the system and it is not the result of an immediate control action as in the classical impedance control scheme [1]. Different examples of implementation of this kind of devices are known in literature, even if limited to one single joint [2], [3], [4], [5], and the application of antagonistic actuated kinematic chains in the field of robotic hand design is under investigation [6].

After a brief review of the dependence of the properties of antagonistic actuation on the transmission elements characteristics, a scheme for simultaneous stiffness-position control of the linearized system is presented. Finally, simulation results of a two-link antagonistic actuated arm are reported and discussed.
\end{abstract}

Index Terms-Antagonistic actuation, variable stiffness mechanisms, feedback linearization, nonlinear systems.

\section{INTRODUCTION}

Standard industrial robots are usually designed to have very rigid links, that implies a considerable increment of the link masses, and to minimize the effects of the elastic coupling between the actuators and the joints due to the deformation of the transmission elements like long shafts, belts or harmonic drives. These design goals are usually maintained also for the design of the control law of these robots. This approach is justified because industrial tasks usually require accuracy, repeatability and simplicity in the implementation of the control law. On the other hand, it is well know that neglecting the elastic coupling between the actuators and the robot joints can lead to vibrations in the kinematic chain and reduce both the dynamic and static performance of the overall system [7], [8], [9], [10].

In the last years, a large variety of robots have been developed to accomplish a completely different class of tasks, like space and submarine activities, cooperative manipulation and, in particular, to interact with humans for entertainment, domestic activities and assistance to elder or handicapped people. The main requirements for the introduction of robots in the human environment are safety and dependability of the robotic system [1], [2], [3]. These requirements exclude the use of standard industrial robots for the interaction with humans. Also incrementing the sensorization and improving the performances of the controller, there are intrinsic limitations on the safety of industrial robots due to the inertia of the links and to the magnitude of the torque that the actuators can apply [3].

The development of lightweight robotic arms [11], [12] is carried out to improve the dynamic performance and reduce the weight-to-payload ratio. While this approach is suitable in case of devices for special applications, in particular for space activities, to improve the safety of robotic arms different projects have been developed, besides maintaining a low level of inertia, introducing also an high compliance at the mechanical level both in the joints of the robot and in the interface between the robot and the environment. Concerning the joint compliance in order to obtain an adequate level of accuracy preserving safety, several variable stiffness devices, and in particular antagonistic actuated joints, have been developed [3], [4], [5]. Continuous high compliant structures with antagonistic actuation are also applied to reduce the mechanical complexity, the weight and the cost of robotic hands [6], [13], [14], [15].

In this paper, the dynamic model of a robot with antagonistic actuated joints similar to the one reported in [3] but without direct coupling between the antagonistic actuators is presented, and the problem of full linearization via static state feedback of both the position and the stiffness of the joint simultaneously is analyzed. The modulation of the mechanical stiffness of the joint is achieved by using transmission elements with nonlinear relation between the displacement and the actuated force [3], [4], [5]. In particular, the cases of transmission elements with quadratic [4] and exponential [5] force-length characteristic are analyzed as examples of application of this methodology. The time dependence of the functions is omitted for brevity in the sequel of the paper.

\section{DYNAMIC MODEL OF ROBOTS WITH ANTAGONISTIC ACTUATED JOINTS}

In this section, the dynamic model of a robotic arm with $N$ antagonistic actuated joints is reported. Some assumptions have been made to obtain this dynamic model. In particular, we assume that the actuators have uniform mass distribution and center of mass on the rotation axis [16] and that their 
rotor kinetic energy is due only to their spinning angular velocity [9]. Another assumption is that each joint is independently actuated by 2 motors in an antagonistic configuration. From now, we refer to this kind of mechanical structure as fully antagonistic actuated kinematic chain. Applications of this methodology to mechanical structures with coupled antagonistic actuation (or non-fully antagonistic, to distinguish from the former case) [6], [15] are object of future research.

The considered model is then composed by $3 N$ rigid bodies ( $N$ links and $2 N$ actuators) with nonlinear elastic coupling between their positions. The state dimension of the model of a robot with $N$ spatial DOFs is then equal to $6 N$ (position and velocity of each rigid body) while the input dimension is $2 N$ (the torques commanded to the actuators). In this case it is necessary to distinguish between the spatial and the stiffness DOFs. The former is the possibility of modifying the position of the system while the latter means the possibility of adjusting the mechanical stiffness of the device. In this terms, a robot with $N$ antagonistic actuated joints ${ }^{1}$ has a total of $2 N$ DOFs ( $N$ spatial $+N$ stiffness).

It is important to stress the fact that, for mechanical stiffness, we mean the compliance of the mechanical coupling between the link and the actuation. Usually this characteristic is imposed by the mechanical design, and in particular by the elasticity of transmission elements, while in this case it can be modulated with antagonistic actuation and nonlinear transmission elements. This allows to increase the safety of the robot arm in the case of unexpected collision with i.e. a human operator. In [2] a meaningful analysis of how the mechanical coupling between the link and the actuation affects the safety of a robotic arm operating in the human environment is reported.

As output of the system, the positions of the joints and of the actuators are considered, obtaining a output of dimension equal to $3 N$.

In Fig. 1 a sketch of a robotic arm with antagonistic actuated joints is reported. With reference to this figure, $\alpha_{i}$ and $\theta_{i}(i=1, \ldots, N)$ are positive in the counterclockwise direction while $\beta_{i}$ is positive in clockwise direction. $\phi_{i}=$ $\phi_{i}\left(\alpha_{i}, \theta_{i}\right)$ and $\psi_{i}=\psi_{i}\left(\beta_{i}, \theta_{i}\right)$ are the nonlinear coupling functions between the position of the joint and of the two actuators (see also Fig. 2).

The dynamic model of the robot with antagonistic actuated joints can be obtained form the standard Lagrangian formulation and can be written in a convenient form grouping the dynamics of the joints and considering separately the dynamics of the two groups of actuators:

$$
\begin{aligned}
\boldsymbol{M}(\boldsymbol{\theta}) \ddot{\boldsymbol{\theta}}+\boldsymbol{C}(\boldsymbol{\theta}, \dot{\boldsymbol{\theta}}) \dot{\boldsymbol{\theta}}+\boldsymbol{D} \dot{\boldsymbol{\theta}}+\boldsymbol{g}_{f}(\boldsymbol{\theta})+\boldsymbol{\varphi}(\boldsymbol{\alpha}, \boldsymbol{\beta}, \boldsymbol{\theta}) & =0 \\
\boldsymbol{J} \ddot{\boldsymbol{\alpha}}+\boldsymbol{B} \dot{\boldsymbol{\alpha}}+\boldsymbol{\phi}(\boldsymbol{\alpha}, \boldsymbol{\theta}) & =\boldsymbol{\tau}_{\alpha} \\
\boldsymbol{J} \ddot{\boldsymbol{\beta}}+\boldsymbol{B} \dot{\boldsymbol{\beta}}+\boldsymbol{\psi}(\boldsymbol{\beta}, \boldsymbol{\theta}) & =\boldsymbol{\tau}_{\beta}
\end{aligned}
$$

where $\boldsymbol{\theta}$ is the vector of joint positions, $\boldsymbol{\alpha}$ and $\boldsymbol{\beta}$ are the vectors of actuator positions, $\boldsymbol{M}(\boldsymbol{\theta})$ is the inertia matrix of the robot, $\boldsymbol{J}$ is the matrix of the inertia moments of the actuators, $\boldsymbol{C}(\boldsymbol{\theta}, \dot{\boldsymbol{\theta}})$ is the matrix of the centrifugal and

\footnotetext{
${ }^{1}$ For mechanical structures with coupled antagonistic actuation, the total number of DOFs $N_{s p+s t}$ (spatial + stiffness) is $N<N_{s p+s t}<2 N$.
}

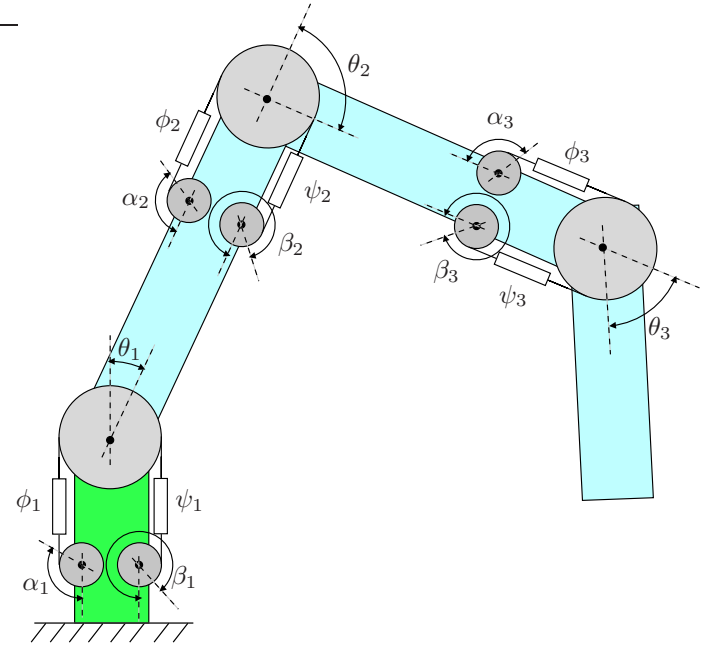

Fig. 1. A robotic arm with 3 antagonistic actuated joints.

Coriolis terms of the robot, $\boldsymbol{D}$ and $\boldsymbol{B}$ are the matrices of the viscous friction coefficients of the robot and of the actuators respectively, $\boldsymbol{g}_{f}(\boldsymbol{\theta})$ is the vector of gravity effects, $\varphi(\boldsymbol{\alpha}, \boldsymbol{\beta}, \boldsymbol{\theta})$ is the combined effect of the positions of the actuators on the joints, $\boldsymbol{\tau}_{\alpha}$ and $\boldsymbol{\tau}_{\beta}$ are the vectors of the torques commanded to the actuators.

The input of the system and the vector of output information are:

$$
\boldsymbol{u}=\left[\begin{array}{c}
\boldsymbol{\tau}_{\alpha} \\
\boldsymbol{\tau}_{\beta}
\end{array}\right], \quad \boldsymbol{y}=\left[\begin{array}{c}
\boldsymbol{\theta} \\
\boldsymbol{\alpha} \\
\boldsymbol{\beta}
\end{array}\right]
$$

Since we are interested to control both the position and the stiffness of the joints of the robot, it is useful to define a new output vector that contains explicitly these information's:

$$
\boldsymbol{y}_{c}=\left[\begin{array}{c}
\theta_{1} \\
\vdots \\
\theta_{N} \\
\frac{\partial \varphi_{1}\left(\alpha_{1}, \beta_{1}, \theta_{1}\right)}{\partial \theta_{1}} \\
\vdots \\
\frac{\partial \varphi_{N}\left(\alpha_{N}, \beta_{N}, \theta_{N}\right)}{\partial \theta_{N}}
\end{array}\right]=\left[\begin{array}{c}
\boldsymbol{\theta} \\
\boldsymbol{S}
\end{array}\right]
$$

where $S$ is the vector of the mechanical stiffness of the joints that can be expressed as in eq.(5) because we suppose all the joints and all the actuators to be independent (no coupling between the movements of the joints or the actuators). From eq. (5) it is possible to note that, in general, while it is difficult to measure the stiffness of the joints, a suitable knowledge of the coupling functions $\varphi(\alpha, \beta, \theta)$ enables us to derive the joints stiffness $\boldsymbol{S}$ from the measured output $\boldsymbol{y}$.

Considering the new output vector $\boldsymbol{y}_{c}$, the input $\boldsymbol{u}$ and the state vector

$$
x=\left[\begin{array}{c}
\theta \\
\dot{\theta} \\
\alpha \\
\dot{\alpha} \\
\beta \\
\dot{\beta}
\end{array}\right]
$$


the model of the robot with antagonistic actuated joints described by eq. (1)-(3) can be rewritten in the input-affine state space form:

$$
\begin{aligned}
\dot{\boldsymbol{x}} & =\boldsymbol{f}(\boldsymbol{x})+\boldsymbol{g}(\boldsymbol{x}) \boldsymbol{u} \\
\boldsymbol{y}_{c} & =\boldsymbol{h}(\boldsymbol{x})
\end{aligned}
$$

where $\boldsymbol{x} \in \mathbb{R}^{6 N}$ and $\boldsymbol{u}, \boldsymbol{y}_{c} \in \mathbb{R}^{2 N}$. In particular, for the model of the robot with antagonistic actuated joints:

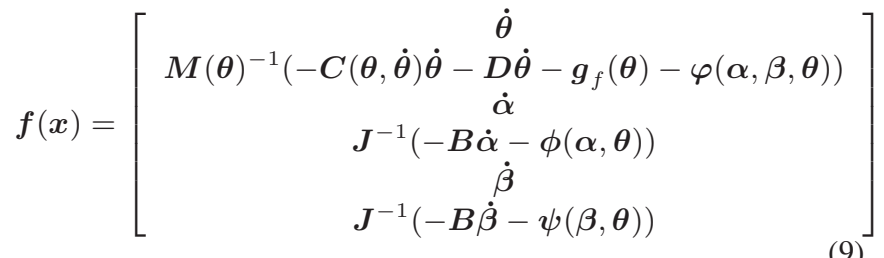

$$
\begin{aligned}
& \boldsymbol{g}(\boldsymbol{x})=\left[\begin{array}{cc}
0_{N \times N} & 0_{N \times N} \\
0_{N \times N} & 0_{N \times N} \\
0_{N \times N} & 0_{N \times N} \\
\boldsymbol{J}^{-1} & 0_{N \times N} \\
0_{N \times N} & 0_{N \times N} \\
0_{N \times N} & \boldsymbol{J}^{-1}
\end{array}\right] \\
& \boldsymbol{h}(\boldsymbol{x})=\left[\begin{array}{l}
\boldsymbol{\theta} \\
\boldsymbol{S}
\end{array}\right]
\end{aligned}
$$

The eq. (7)-(11) define a square nonlinear system that can be linearized via static feedback if suitable conditions are satisfied [17], [18].

\section{Static FEEDBACK LinEARIZATION}

In this section, necessary and sufficient conditions for the solution of the problem of full linearization via static state feedback of the system described by the eq. (1)-(5) are analyzed [17]. In particular, we have to check if the decoupling matrix of this system is nonsingular and if the sum of the relative degrees of the outputs are equal to the state dimension of the system.

With the aim of simplifying the explanation, the notation used in the previous section is extended. Since all the components of both the input, the output and the state vectors belong to $\mathbb{R}^{N}$, where $N$ is the number of robot joints, we can redefine the dimensions of the input $\boldsymbol{u}$, the output $\boldsymbol{y}_{c}$ and the state $\boldsymbol{x}$ of the system to 2, 2 and 6, respectively. Also the notation of Lie derivative is extended to work with vectors in $\mathbb{R}^{N}$.

The problem of static feedback linearization consists in transforming a nonlinear system of the type (7)-(8), via static state feedback and coordinate transformations into a fully controllable and observable linear system that can be represented in the form:

$$
\dot{\boldsymbol{z}}=\boldsymbol{A} \boldsymbol{z}+\boldsymbol{B}\left(\left[\begin{array}{c}
L_{f}^{r_{1}} h_{1}\left(\boldsymbol{\Phi}^{-1}(\boldsymbol{z})\right) \\
\vdots \\
L_{f}^{r_{m}} h_{m}\left(\boldsymbol{\Phi}^{-1}(\boldsymbol{z})\right)
\end{array}\right]+\boldsymbol{Q}\left(\boldsymbol{\Phi}^{-1}(\boldsymbol{z})\right) \boldsymbol{u}\right)
$$

$\boldsymbol{y}_{c}=\boldsymbol{C z}$

where $\boldsymbol{A}, \boldsymbol{B}$ and $\boldsymbol{C}$ are matrices of proper dimensions given by the Brunowsky canonical form, $m$ is the number outputs of the system, $r_{1}, \ldots, r_{m}$ are the relative degrees of each output, $L_{f} h(x)$ denotes the Lie derivative of $\boldsymbol{h}(\boldsymbol{x})$ along the vector function $\boldsymbol{f}(\boldsymbol{x}), \boldsymbol{z}=\boldsymbol{\Phi}(\boldsymbol{x})$ is the coordinate transformation from the original to the new state space and $\boldsymbol{Q}(\boldsymbol{x})$ is the so-called decoupling matrix.

First of all, we have to define the (vector) relative degree of the outputs of the system. For this purpose, it is useful to firstly define the state transformation from the original state space to the state space of the linearized system:

$$
\boldsymbol{\Phi}(\boldsymbol{x})=\left[\begin{array}{c}
h_{1}(\boldsymbol{x}) \\
\vdots \\
L_{f}^{\left(r_{1}-1\right)} h_{1}(\boldsymbol{x}) \\
\vdots \\
h_{m}(\boldsymbol{x}) \\
\vdots \\
L_{f}^{\left(r_{m}-1\right)} h_{m}(\boldsymbol{x})
\end{array}\right]
$$

The relative vector degree of the outputs can now be easily found by looking when the inputs appearing explicitly in eq. (14). In particular, for the output $\boldsymbol{\theta}$ it possible to find that:

$$
\begin{aligned}
L_{g_{(\alpha, \beta)}} L_{f}^{k} h_{\theta}(\boldsymbol{x}) & =0_{N \times N} \quad, \quad i=0, \cdots, 2 \\
L_{g_{\alpha}} L_{f}^{3} h_{\theta}(\boldsymbol{x}) & =\boldsymbol{J}^{-1} \boldsymbol{M}(\boldsymbol{\theta})^{-1} \frac{\partial \boldsymbol{\varphi}(\boldsymbol{\alpha}, \boldsymbol{\beta}, \boldsymbol{\theta})}{\partial \boldsymbol{\alpha}} \\
L_{g_{\beta}} L_{f}^{3} h_{\theta}(\boldsymbol{x}) & =\boldsymbol{J}^{-1} \boldsymbol{M}(\boldsymbol{\theta})^{-1} \frac{\partial \boldsymbol{\varphi}(\boldsymbol{\alpha}, \boldsymbol{\beta}, \boldsymbol{\theta})}{\partial \boldsymbol{\beta}}
\end{aligned}
$$

where $h_{\theta}(\boldsymbol{x})$ denotes the restriction of the output vector to $\boldsymbol{\theta}$ only while for the output $\boldsymbol{S}$ :

$$
\begin{aligned}
& L_{g_{(\alpha, \beta)}} h_{S}(\boldsymbol{x})=0_{N \times N} \\
& L_{g_{\alpha}} L_{f} h_{S}(\boldsymbol{x})=\boldsymbol{J}^{-1} \frac{\partial \boldsymbol{S}}{\partial \boldsymbol{\alpha}} \\
& L_{g_{\beta}} L_{f} h_{S}(\boldsymbol{x})=\boldsymbol{J}^{-1} \frac{\partial \boldsymbol{S}}{\partial \boldsymbol{\beta}}
\end{aligned}
$$

where $h_{S}(\boldsymbol{x})$ denotes the restriction of the output vector to its component $S$. $L_{g_{\alpha}}$ and $L_{g_{\beta}}$ denote the restriction of the Lie derivative to the $\tau_{\alpha}$ and $\tau_{\beta}$ component of the input vector respectively while $L_{g_{(\alpha, \beta)}}$ denotes both these cases.

From this result we can state that, if the derivatives of $\boldsymbol{\varphi}(\boldsymbol{\alpha}, \boldsymbol{\beta}, \boldsymbol{\theta})$ in eq. (16),(17),(19),(20) are not null, the vector relative degree of $\boldsymbol{\theta}$ is 4 while the one of $\boldsymbol{S}$ is 2 . The sum of the vector relative degrees of the output is then equal to the dimension of the state of the system (i.e. 6), so that the condition for the existence of noninteracting control via static state feedback is satisfied. In particular, if $\boldsymbol{\varphi}(\boldsymbol{\alpha}, \boldsymbol{\beta}, \boldsymbol{\theta})$ depends linearly on their arguments, as in [7], [9], [10], [19], the terms in eq.(19),(20), and also the successive derivatives, are always zero. Hence the vector relative degree of $S$ is not defined and therefore the mechanical stiffness of the joints is not controllable.

Now, to verify that the system has no zero dynamics, we have to check if the decoupling matrix is nonsingular. The decoupling matrix of this system is:

$$
\boldsymbol{Q}(\boldsymbol{x})=\boldsymbol{B}(\boldsymbol{\theta})\left[\begin{array}{cc}
\frac{\partial \varphi(\boldsymbol{\alpha}, \boldsymbol{\beta}, \boldsymbol{\theta})}{\partial \boldsymbol{\alpha}} & \frac{\partial \varphi(\boldsymbol{\alpha}, \boldsymbol{\beta}, \boldsymbol{\theta})}{\partial \boldsymbol{\beta}} \\
\frac{\partial \boldsymbol{S}}{\partial \boldsymbol{\alpha}} & \frac{\partial \boldsymbol{S}}{\partial \boldsymbol{\beta}}
\end{array}\right]
$$


where

$$
\boldsymbol{B}(\boldsymbol{\theta})=\left[\begin{array}{cc}
\boldsymbol{J}^{-1} \boldsymbol{M}(\boldsymbol{\theta})^{-1} & 0_{N \times N} \\
0_{N \times N} & \boldsymbol{J}^{-1}
\end{array}\right]
$$

From this equation and from eq.(5), it is possible to see that the rank of $\boldsymbol{Q}(\boldsymbol{x})$ depends on the nature of $\varphi(\boldsymbol{\alpha}, \boldsymbol{\beta}, \boldsymbol{\theta})$, hence this property has to be checked for mechanical implementation. If $\varphi(\boldsymbol{\alpha}, \boldsymbol{\beta}, \boldsymbol{\theta})$ depends linearly on their arguments, $\boldsymbol{S}$ is constant and therefore $\boldsymbol{Q}$ is singular.

Now, supposing that $\boldsymbol{Q}(\boldsymbol{x})$ is not singular, by defining the new input

$$
\boldsymbol{u}=\boldsymbol{Q}^{-1}(\boldsymbol{x})\left(\left[\begin{array}{l}
-L_{f}^{4} h_{\theta}(\boldsymbol{x}) \\
-L_{f}^{2} h_{S}(\boldsymbol{x})
\end{array}\right]+\left[\begin{array}{c}
\boldsymbol{v}_{\theta} \\
\boldsymbol{v}_{S}
\end{array}\right]\right)
$$

we obtain the linear model of the robot with elastic joints

$$
\begin{aligned}
\dot{z} & =\boldsymbol{A} \boldsymbol{z}+\boldsymbol{B} \boldsymbol{v}, \quad \boldsymbol{v}=\left[\begin{array}{c}
\boldsymbol{v}_{\theta} \\
\boldsymbol{v}_{S}
\end{array}\right] \\
\boldsymbol{y}_{c} & =\boldsymbol{C} \boldsymbol{z}
\end{aligned}
$$

where

$$
\begin{aligned}
& \boldsymbol{A}=\left[\begin{array}{llllll}
0 & I & 0 & 0 & 0 & 0 \\
0 & 0 & I & 0 & 0 & 0 \\
0 & 0 & 0 & I & 0 & 0 \\
0 & 0 & 0 & 0 & 0 & 0 \\
0 & 0 & 0 & 0 & 0 & I \\
0 & 0 & 0 & 0 & 0 & 0
\end{array}\right], \quad \boldsymbol{B}=\left[\begin{array}{ll}
0 & 0 \\
0 & 0 \\
0 & 0 \\
I & 0 \\
0 & 0 \\
0 & I
\end{array}\right] \\
& \boldsymbol{C}=\left[\begin{array}{llllll}
I & 0 & 0 & 0 & 0 & 0 \\
0 & 0 & 0 & 0 & I & 0
\end{array}\right] \\
& \boldsymbol{z}=\left[\begin{array}{llllll}
\boldsymbol{\theta}^{T} & \dot{\boldsymbol{\theta}}^{T} & \ddot{\boldsymbol{\theta}}^{T} & \boldsymbol{\theta}^{[3] T} & \boldsymbol{S}^{T} & \dot{\boldsymbol{S}}^{T}
\end{array}\right]^{T}
\end{aligned}
$$

in which $I$ and 0 are the identity and the zero matrix of dimension $N$. From eq. (24)-(28) it is then possible to note that:

$$
\left[\begin{array}{c}
\boldsymbol{\theta}^{[4]} \\
\ddot{\boldsymbol{S}}
\end{array}\right]=\left[\begin{array}{c}
\boldsymbol{v}_{\theta} \\
\boldsymbol{v}_{S}
\end{array}\right]
$$

\section{Control strategy}

Since the system (24),(25) is completely controllable and observable, the state of the system can be reconstructed by means of an asymptotic observer and of the change of coordinates (14) or, since the position of each rigid body is directly measurable, the velocities can be estimated in many ways e.g. by means of state variable filters or adaptive windowing algorithms [20].

From eq.(29), applying the control laws:

$$
\begin{aligned}
v_{\theta}= & \boldsymbol{\theta}_{d}^{[4]}+\boldsymbol{K}_{3_{\theta}}\left(\theta_{d}^{[3]}-L_{f}^{3} h_{\theta}(\boldsymbol{x})\right)+\boldsymbol{K}_{2_{\theta}}\left(\ddot{\boldsymbol{\theta}}_{d}-L_{f}^{2} h_{\theta}(\boldsymbol{x})\right)+\ldots \\
& \ldots \boldsymbol{K}_{1_{\theta}}\left(\dot{\boldsymbol{\theta}}_{d}-L_{f} h_{\theta}(\boldsymbol{x})\right)+\boldsymbol{K}_{0_{\theta}}\left(\boldsymbol{\theta}_{d}-h_{\theta}(\boldsymbol{x})\right) \\
v_{S}= & \ddot{\boldsymbol{S}}_{d}+\boldsymbol{K}_{1_{S}}\left(\dot{\boldsymbol{S}}_{d}-L_{f} h_{S}(\boldsymbol{x})\right)+\boldsymbol{K}_{0_{S}}\left(\boldsymbol{S}_{d}-h_{S}(\boldsymbol{x})\right)
\end{aligned}
$$

with diagonal gain matrices $\boldsymbol{K}_{3_{\theta}}, \ldots, \boldsymbol{K}_{0_{S}}$ such that

$$
\begin{gathered}
\lambda^{4}+\lambda^{3} \boldsymbol{K}_{3_{\theta_{i}}}+\lambda^{2} \boldsymbol{K}_{2_{\theta_{i}}}+\lambda \boldsymbol{K}_{1_{\theta_{i}}}+\boldsymbol{K}_{0_{\theta_{i}}}=0, i=1, \ldots, n \\
\lambda^{2}+\lambda \boldsymbol{K}_{1_{S_{i}}}+\boldsymbol{K}_{0_{S_{i}}}=0, i=1, \ldots, n
\end{gathered}
$$

are Hurwitz polynomials, the convergence to zero of the tracking error is ensured. If the desired trajectory $\boldsymbol{\theta}_{d}$ is continuous together with its derivatives up to the 4 th order and $\boldsymbol{S}_{d}$ is continuous together with its derivatives up to the 2nd, also the asymptotic trajectory tracking is achieved.

It is important to note that all the Lie derivative appearing in eq. (23),(30) and (31) can be written as function of the measurable quantities $x$, so the computation of the time derivatives of $\boldsymbol{\theta}$ (up to 4 th order) and $\boldsymbol{S}$ (up to 2 nd order) are not necessary.

The control law in eq. (30),(31) is equivalent to a static state feedback plus feedforward in the state space of the linearized system:

$$
\boldsymbol{v}=\boldsymbol{v}_{d}+\boldsymbol{K}\left(\boldsymbol{z}_{d}-\boldsymbol{\Phi}(\boldsymbol{x})\right)
$$

$$
\boldsymbol{v}_{d}=\left[\begin{array}{c}
\boldsymbol{\theta}_{d}^{[4]} \\
\ddot{\boldsymbol{S}}_{d}
\end{array}\right] \quad, \quad \boldsymbol{z}_{d}=\left[\begin{array}{c}
\boldsymbol{\theta}_{d} \\
\dot{\boldsymbol{\theta}}_{d} \\
\ddot{\boldsymbol{\theta}}_{d} \\
\boldsymbol{\theta}_{d}^{[3]} \\
\boldsymbol{S}_{d} \\
\dot{\boldsymbol{S}}_{d}
\end{array}\right]
$$

$$
\boldsymbol{K}=\operatorname{diag}\left[\begin{array}{llllll}
\boldsymbol{K}_{0_{\theta}} & \boldsymbol{K}_{1_{\theta}} & \boldsymbol{K}_{2_{\theta}} & \boldsymbol{K}_{3_{\theta}} & \boldsymbol{K}_{0_{S}} & \boldsymbol{K}_{1_{S}}
\end{array}\right]
$$

The matrix $\boldsymbol{K}$ can be also obtained via direct eigenvalues assignment or through the solution of the CARE equation with a suitable choice of the weight matrices.

This approach ensures that, in case of an undesired event e. g. a collision with an obstacle, the coupling between the joint and the actuators has the desired stiffness without the intervention of the controller, avoiding in this way any problem related to limited control bandwidth, sensorization, delay in the control loop and so on. If the stiffness $S$ depends itself on $\boldsymbol{\theta}$, this behavior is a linearization of the system stiffness around the desired position $\boldsymbol{\theta}_{d}$.

\section{PROPERTIES OF THE TRANSMISSION ELEMENTS}

To show some properties of different types of nonlinear coupling between the joint and the actuators, transmission elements with quadratic and exponential relation between the displacement and the force will be investigated. This analysis is performed considering a two-links planar arm with antagonistic actuated joints. In Fig. 2 a detail of the antagonistic joint is given. From this picture, the displacement between the $i$-th joint and its actuators can be defined as:

$$
\begin{aligned}
\epsilon_{\alpha_{i}} & =r_{m} \alpha_{i}+r_{j} \theta_{i} \\
\epsilon_{\beta_{i}} & =r_{m} \beta_{i}-r_{j} \theta_{i}
\end{aligned}
$$

where $\epsilon_{\alpha_{i}}, \epsilon_{\beta_{i}}$ are the displacements (compression or extension) of the transmission elements and $r_{m}, r_{j}$ are the radii of the pulley of the actuator and of the joint respectively. In order to simplify the following calculations and without loss of generality, $r_{m}$ and $r_{j}$ are supposed to be equals for all the actuators and all the joints. In [4], [5] the transmission elements are connected to the actuators and to the joint by means of metallic cables. To avoid cable slack, a suitable operating region of $\boldsymbol{\alpha}, \boldsymbol{\beta}, \boldsymbol{\theta}$ must be defined considering the geometry of the device. However, the condition $\epsilon_{\alpha_{i}, \beta_{i}}<0$ can be achieved by properly setting the zero position of the 
actuators. From Fig. 2 it is also possible to establish the relationship between the coupling functions of the joint and of the actuators:

$$
\varphi_{i}\left(\alpha_{i}, \beta_{i}, \theta_{i}\right)=\frac{r_{j}}{r_{m}}\left[\phi_{i}\left(\alpha_{i}, \theta_{i}\right)-\psi_{i}\left(\beta_{i}, \theta_{i}\right)\right]
$$

\section{A. Quadratic force-displacement transmission elements}

In order to provide a stiffness that is a linear function of the displacement and independent from the position of the joint, transmission elements with quadratic forcecompression characteristic must be used [4]:

$$
F=k_{2} \epsilon^{2}+k_{1} \epsilon+k_{0}
$$

where $F$ is the force, $\epsilon$ is the displacement and $k_{2,1,0}$ are proper constants. The torques applied to the actuators result:

$$
\begin{aligned}
& \phi_{i}\left(\alpha_{i}, \theta_{i}\right)=r_{m}\left(k_{2} \epsilon_{\alpha_{i}}^{2}+k_{1} \epsilon_{\alpha_{i}}+k_{0}\right) \\
& \psi_{i}\left(\beta_{i}, \theta_{i}\right)=r_{m}\left(k_{2} \epsilon_{\beta_{i}}^{2}+k_{1} \epsilon_{\beta_{i}}+k_{0}\right)
\end{aligned}
$$

and the torque applied to the $i$-th joint is:

$$
\begin{gathered}
\varphi_{i}\left(\alpha_{i}, \beta_{i}, \theta_{i}\right)=r_{j}\left[k_{2}\left(\epsilon_{\alpha_{i}}^{2}-\epsilon_{\beta_{i}}^{2}\right)+k_{1}\left(\epsilon_{\alpha_{i}}-\epsilon_{\beta_{i}}\right)\right]= \\
\quad=r_{j}\left[k_{2} r_{m}\left(\alpha_{i}+\beta_{i}\right)+k_{1}\right]\left[r_{m}\left(\alpha_{i}-\beta_{i}\right)+2 r_{j} \theta_{i}\right]
\end{gathered}
$$

Deriving this expression with respect to $\theta_{i}$ the value of the joint stiffness is obtained:

$$
\frac{\partial \varphi_{i}\left(\alpha_{i}, \beta_{i}, \theta_{i}\right)}{\partial \theta_{i}}=S_{i}=2 r_{j}^{2}\left[k_{2} r_{m}\left(\alpha_{i}+\beta_{i}\right)+k_{1}\right]
$$

In this case, the joint stiffness does not depend on the position of the joint: this result can be achieved only with quadratic force-displacement relation as eq. (40). The decoupling matrix for the two-links planar arm can be be written as:

$$
\begin{aligned}
& \boldsymbol{Q}(\boldsymbol{x})=r_{j} r_{m} \boldsymbol{B}(\boldsymbol{\theta})\left[\begin{array}{ccl}
\left(2 k_{2} \epsilon_{\alpha_{1}}+k_{1}\right) & 0 & \\
0 & \left(2 k_{2} \epsilon_{\alpha_{2}}+k_{1}\right) & \\
2 k_{2} r_{j} & 0 & \ldots \\
0 & 2 k_{2} r_{j} &
\end{array}\right.
\end{aligned}
$$

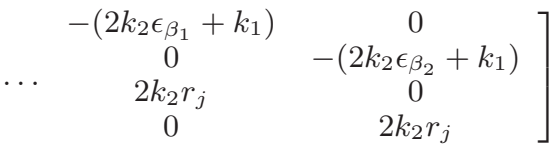

In this case, $\boldsymbol{Q}(\boldsymbol{x})$ is singular if $\epsilon_{\alpha_{i}}=0$ or $\epsilon_{\beta_{i}}=0$ and $k_{1}=0$ (purely quadratic force-displacement characteristic, zero stiffness for zero displacement) and is always singular if $k_{2}=0$, confirming that the stiffness of the joints is not adjustable using transmission elements with linear compliance. Another condition of singularity of $\boldsymbol{Q}(\boldsymbol{x})$ :

$$
\alpha_{i}+\beta_{i}=-\frac{k_{1}}{k_{2} r_{m}}
$$

correspond, from eq. (44), to the case in which $S_{i}=0$, that means no coupling between the actuators and the joint, a nonsense. This condition can be avoided imposing a suitable minimum value of the stiffness.

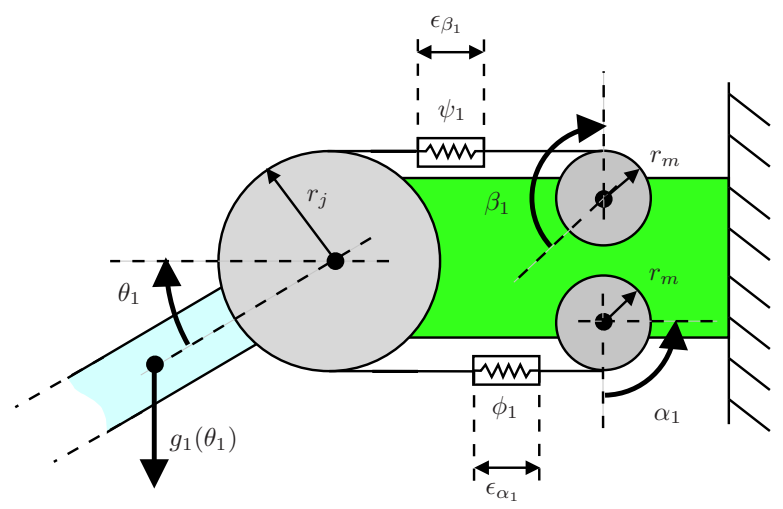

Fig. 2. Detail of the antagonistic actuated joint.

\section{B. Exponential force-displacement transmission elements}

In this example, the transmission elements are composed by plastic elements (gum-balls) compressed inside a cylinder [5]. The geometry and the characteristic of the material gives the nonlinear force-compression relation [21], [22], [23]:

$$
F=k\left(e^{b \epsilon}-1\right)
$$

where $F$ is the force, $\epsilon$ is the displacement and $k>0, b>0$ are suitable constants. The torques applied to the actuators of the $i$-th joint are then:

$$
\begin{aligned}
& \phi_{i}\left(\alpha_{i}, \theta_{i}\right)=r_{m} k\left[e^{b \epsilon_{\alpha_{i}}}-1\right] \\
& \psi_{i}\left(\beta_{i}, \theta_{i}\right)=r_{m} k\left[e^{b \epsilon_{\beta_{i}}}-1\right]
\end{aligned}
$$

The resulting torque applied to the joint using these elements in antagonistic configuration is:

$$
\varphi_{i}\left(\alpha_{i}, \beta_{i}, \theta_{i}\right)=r_{j} k\left[e^{b \epsilon_{\alpha_{i}}}-e^{b \epsilon_{\beta_{i}}}\right]
$$

The resulting stiffness of the joint can be then expressed in the form:

$$
\frac{\partial \varphi_{i}\left(\alpha_{i}, \beta_{i}, \theta_{i}\right)}{\partial \theta_{i}}=S_{i}=k b r_{j}^{2}\left[e^{b \epsilon_{\alpha_{i}}}+e^{b \epsilon_{\beta_{i}}}\right]
$$

The decoupling matrix for the two-links planar arm can be be written as:

$Q(\boldsymbol{x})=$

$k b r_{j} r_{m} \boldsymbol{B}(\boldsymbol{\theta})\left[\begin{array}{cccc}e^{b \epsilon_{\alpha_{1}}} & 0 & -e^{b \epsilon_{\beta_{1}}} & 0 \\ 0 & e^{b \epsilon_{\alpha_{2}}} & 0 & -e^{b \epsilon_{\beta_{2}}} \\ b r_{j} e^{b \epsilon_{\alpha_{1}}} & 0 & b r_{j} e^{b \epsilon_{\beta_{1}}} & 0 \\ 0 & b r_{j} e^{b \epsilon_{\alpha_{2}}} & 0 & b r_{j} e^{b \epsilon_{\beta_{2}}}\end{array}\right]$ (52)

It is important to note that in this case $\boldsymbol{Q}(\boldsymbol{x})$ is always nonsingular thanks to the properties of the exponential function that characterize the transmission elements.

\section{Simulation of the TWO-Link Antagonistic ACTUATED ARM}

In the following the validity of the proposed approach is reported by presenting the simulation results of a planar twolink antagonistic actuated arm. Due to space limitations, the well-known model of the planar two-link arm together with the solution of the previous equations for this system are omitted. For the same reason, only the simulations about the 
exponential force-displacement transmission elements case are reported.

In the simulation scheme, only the joint and actuator positions are known and the corresponding velocities are estimated by means of state variable filters. The trajectories are generated through filters of appropriate order to estimate also their derivatives up to the necessary order. The control strategy has been chosen as in eq. (35) and the matrix $\boldsymbol{K}$ is obtained from the solution of the CARE equation with a diagonal state weights matrix.

In Fig. 3 the positions of the joints of the antagonistic actuated arm are reported. Both step and sinusoidal joint trajectories are used together with coordinated movements to show the stabilizing properties of the controller. The joint stiffnesses and the stiffness errors are reported in Fig.4. During the test, the stiffness of the joint 2 is constant while the one of joint 1 is sinusoidal. It is important to note that the joint stiffness trajectories are not affected by the changes of the joint positions and vice versa.

\section{CONCLUSIONS AND FUTURE WORK}

In this paper, the non-interacting static feedback linearization of a antagonistic actuated arm has been presented. This problem can be solved if the transmission elements have nonlinear force-displacement characteristic and if the transmission element stiffness with zero deformation $S_{0} \neq 0$.

The simultaneous stiffness-position control of a two-link robot arm has been validated through simulation. Even if only the joint and the actuator positions are known, the velocities estimation through state variable filters does not compromise the stability of the closed loop system.

Future activities consist in the implementation of this control scheme on a single link experimental setup [5] and the extension of the proposed approach to the case of nonlinear visco-elastic transmission elements.

The problem of external load compensation for this system is under analysis while the extension of this approach to nonfully antagonistic actuated robots is object of future research.

\section{REFERENCES}

[1] A. Bicchi, S. Lodi Rizzini, and G. Tonietti. Compliant design for intrinsic safety: General issue and preliminariey design. In Proc. of IEEE/RSJ Int. Conf. on Intelligent Robots and Systems, 2001.

[2] A. Bicchi and G. Tonietti. Fast and soft arm tactics: Dealing with the safety-performance trade-off in robot arms design and control. IEEE Robotics and Automation Magazine, 11(2):22-33, 2004.

[3] G. Tonietti, R. Schiavi, and A. Bicchi. Design and control of a variable stiffness actuator for safe and fast physical human/robot interaction. In Proc. IEEE Int. Conf. on Robotics and Automation, pages 528-533, 2005.

[4] S. A. Migliore, E. A. Brown, and S. P. DeWeerth. Biologically inspired joint stiffness control. In Proc. IEEE Int. Conf. on Robotics and Automation, 2005.

[5] G. Palli, C. Melchiorri, T. Wimböck, M. Grebenstein, and G. Hirzinger. Experiments with the DLR's antagonistic actuated joint. In Submitted to IFAC Trans. on MECHATRONICS, 2007.

[6] F. Lotti and G. Vassura. A novel approach to mechanical design of articulated finger for robotic hands. In Proc. IEEE/RSJ IROS Int. Conf. on Intelligent Robot and Systems, 2002.

[7] A. De Luca. Dynamic control of robots with joint elasticity. In Proc. IEEE Int. Conf. on Robotics and Automation, pag. 152-158 vol.1, 1988.
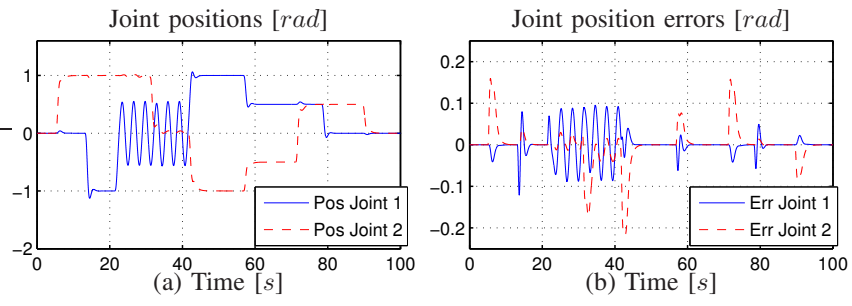

Fig. 3. (a) Joint positions and (b) trajectory tracking errors
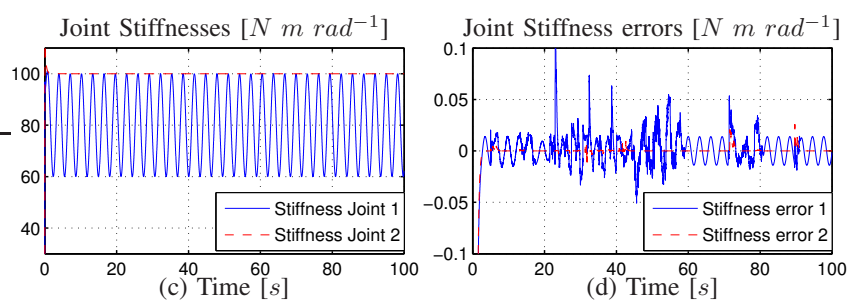

Fig. 4. (c) Joint stiffnesses and (d) stiffness tracking errors

[8] M. Vukobratovi, V. Matijevi, and V. Potkonjak. Control of robots with elastic joints interacting with dynamic environment. J. Intell. Robotics Syst., 23(1):87-100, 1998.

[9] M. W. Spong. Modeling and control of elastic joint robots. Journal of Dynamic Systems, Measurement, and Control, 109(4):310-319, 1987.

[10] A. De Luca and P. Lucibello. A general algorithm for dynamic feedback linearization of robots with elastic joints. In Proc. IEEE Int. Conf. on Robotics and Automation, pages 504-510 vol.1, 1998.

[11] G. Hirzinger, A. Albu-Schäffer, M. Hähnle, I. Schäfer, and N. Sporer On a new generation of torque controlled light-weight robots. In Proc. IEEE Int. Conf. on Robotics and Automation, volume 4, pages 33563363, 2001.

[12] G. Hirzinger, N. Sporer, A. Albu-Schäffer, M. Hahnle, R. Krennand A. Pascucci, and M. Schedl. DLR's torque-controlled light weight robot III - Are we reaching the technological limits now? In Proc. IEEE Int. Conf. on Robotics and Automation, pages 1710-1716, 2002.

[13] L. Biagiotti, F. Lotti, C. Melchiorri, P. Tiezzi, and G. Vassura. UBH 3: an anthropomorphic hand with simplied endo-skeletal structure and soft continuous fingerpads. In Proc. IEEE Int. Conf. on Robotics and Automation, 2004.

[14] L. Biagiotti, F. Lotti, C. Melchiorri, G. Palli, P. Tiezzi, and G. Vassura. Development of UB Hand 3: Early results. In Proc. IEEE Int. Conf. on Robotics and Automation, 2005.

[15] G. Palli. Model and control of anthropomorphic robotic hands through visual servoing. Master's thesis, University of Bologna, Dept. of Electronic, Computer Science ans Systems, 2003. (in italian).

[16] P. Tomei. A simple PD controller for robots with elastic joints. In IEEE Trans. on Automatic Control, volume 36, pages 1208-1213, 1991.

[17] A. Isidori. Nonlinear Control Systems, 3rd edition. Springer-Verlag New York, Inc., 1995.

[18] A. De Luca and P. Tomei. Theory of Robot Control, chapter Elastic Joints. Springer-Verlag New York, Inc., 1996.

[19] A. De Luca and L. Lanari. Robots with elastic joints are linearizable via dynamic feedback. In Proc. 34th IEEE Int. Conf. Decision and Control, pages 3895-3897 vol.4, 1995.

[20] Farrokh Janabi-Sharifi, Vincent Hayward, and Chung-Shin J. Chen. Discrete-time adaptive windowing for velocity estimation. IEEE Trans. on Control Systems technology, 8(6):1003+, 2000.

[21] K. H. Hunt and F. R. E. Crossley. Coeffcient of restitution interpreted as damping in vibroimpact. ASME Journal of Appl. Mech., 1975.

[22] C. Melchiorri L. Biagiotti, P. Tiezzi and G. Vassura. Modelling and identification of soft pads for robotic hands. In Proc. IEEE/RSJ Int. Conf. on Intelligent Robots and Systems, 2005.

[23] N. Diolaiti, C. Melchiorri, and S. Stramigioli. Contact impedance estimation for robotic systems. In IEEE Transactions on Robotics, volume 21, 2005. 